
ИНФЕРТИЛЬНЫХ МУЖЧИН

Ниткин.Д. M. (nitkin@tut.by),РакевичM.B. (justmaks@mail.ru), Журко П. T. (PavlinaTS@mail.ru)

ГУО «Белорусская медицинская академия последипломного образования», Минск, Беларусь

Введение. Разработка методов диагностики и лечения мужского бесплодия в настоящее время является актуальной задачей.

Целью работы было определение показателей спермограммы, антиокислительной способности спермоплазмы и концентрации циинка в спермоплазме у здоровых фертильных мужчин и мужчин, страдающчих бесплодием, а такље оценка влияния лекарственных средств с антиоксидантной активностью на параметры спермограммы у инфертильных мужчин.

Материал и методы. В исследовании приняли участие 144 мужчины репродуктивного возраста: 45 фертильных, 99 инфертильных. Сбор и анализ эякулята осуществлялся в соответствии с критериями ВОЗ (2010).

Результаты. Имелись статистически значимые различия в параметрах спермограммы между фертильнылм и инфертильнылм мужчинами $(p<0,05)$. У 48,5\% инфертильных пациентов антиоксидантная активность спермоплазмы находилась в пределах нормы, у 51,5\% - ниже 1,6 ммоль/л. Концентрация ицинка у мужчин с бесплодием определялась на уровне 1,62 [1,07; 2,0] ммоль/л. Применение комплекса антиоксидантов в составе L-карнитина, пентоксифиллина, цинк сульфата, витамина E, фолиевой кислоты сроком на 3 месяца улучшило качественные и количественные характеристики эякулята у инфертильных мужчин $(p<0,05)$, а также общую антиоксидантную активность спермоплазмы $(1,88$ [1,69; 2,03] ммоль/л) и концентрацию цуинка в сперме $(2,08[1,51 ; 2,58]$ ммоль/л).

Bыводы. Снижение общей антиоксидантной активности является фактором развития мужского бесплодия. Назначение комплекса антиоксидантов улучшает качественные и количественные показатели спермограммы.

Ключевые слова: мужское бесплодие, спермограмма, антиоксидантная активность спермоплазмы, лечение бесплодия.

\section{Введение}

В настоящее время от 8 до $15 \%$ супружеских пар репродуктивного возраста сталкиваются с неспособностью зачать ребенка, при этом в половине случаев причина бесплодия - мужской фактор $[1,2]$. Роль мужского фактора в семейном бесплодии по-прежнему недооценивается, его частота увеличивается с каждым годом, при этом возникают трудности с выявлением его причин и эффективностью лечения. Разработка методов диагностики и лечения мужского бесплодия в настоящее время является актуальным вопросом, поскольку от качества проведенных диагностических мероприятий, использования необходимых препаратов, а также рекомендаций по образу жизни и питанию зависит конечный результат - достижение зачатия и рождение здорового ребенка, а этот факт оказывает ключевое влияние на качество жизни супружеской пары.

Наиболее значимыми причинами развития бесплодия у мужчин являются врожденные пороки развития, инфекционно-воспалительные заболевания мочеполовой системы, генетические аномалии, метаболические, эндокринные, прием лекарственных препаратов, факторы внешней среды и другие [2, 3, 4, 5]. В 30-40\% случаев мужского бесплодия имеет место так называемое идиопатическое бесплодие, когда при наличии олиго-, астено-, тератозооспермии отсутствуют анамнестические факторы риска, не выявляются нарушения в результате медицинских осмотров и гормональных исследований $[1,2,5]$. Принято считать, что в $25-40 \%$ случаев идиопатического бесплодия причиной является окислительный стресс, возникающий при дисбалансе между активными формами кислорода (АФК) и антиоксидантной защитной функцией эякулята $[6,7]$. Установлено, что у инфертильных мужчин по сравнению с фертильными в сперме отмечаются более высокие значения АФК $[4,8]$. Избыток АФК в сперме приводит к подавлению сперматогенеза, повреждению мембран сперматозоидов, нарушению процессов капацитации, акросомальной реакции, слияния сперматозоида с яйцеклеткой [3, 9].

Для коррекции показателей спермограммы инфертильных мужчин и уменьшения образования АФК используют лекарственные средства, обладающие антиоксидантными свойствами. Антиоксиданты представляют собой природные или синтетические биомолекулы, которые препятствуют повреждению структуры клеток АФК. Для лечения мужского бесплодия наиболее часто применяют витамины А, Е, C, карнитин, цинк, селен и экстракты растений $[10,11]$. Имея невысокий риск токсичности и низкую стоимость, антиоксиданты рекомендованы для лечения мужского бесплодия Европейской ассоциацией урологов [1].

По результату метаанализа, проведенного Showell M. et al., антиоксиданты улучшают жизнеспособность, концентрацию, прогрессивную подвижность, снижают фрагментацию ДНК сперматозоидов, повышают процент беременностей при естественном зачатии и программах вспомогательных репродуктивных технологий $[12,13]$. 
Однако имеются и другие данные, указывающие на то, что несбалансированный или избыточный прием антиоксидантов может привести к увеличению наличия разрывов в ядерном хроматине сперматозоидов и спонтанным выкидышам на ранних сроках беременности [11].

Целью работы было определение показателей спермограммы, антиокислительной способности спермоплазмы и концентрации цинка в спермоплазме у здоровых фертильных мужчин и мужчин, страдающих бесплодием, а также оценка влияния лекарственных средств с антиоксидантной активностью на параметры спермограммы у инфертильных мужчин.

\section{Материал и методы}

В исследовании приняли участие 144 мужчины репродуктивного возраста. Лабораторные исследования проходили на базе НИЛ БелМАПО, кафедры урологии и нефрологии, кафедры клинической лабораторной диагностики БелМАПО с 2015 по 2017 гг. Все мужчины на момент проведения испытаний постоянно проживали на территории Республики Беларусь, медиана возраста составила 31 [27; 34] год.

Основным критерием отбора для включения в группу фертильных мужчин $(\mathrm{n}=45)$ было наличие 1 и более ребенка, в группу инфертильных мужчин $(\mathrm{n}=99)$ - жалобы на бесплодие в браке более 1 года и наличие патоспермии. Критерии исключения: тяжелая соматическая патология и врожденные пороки развития органов мочеполовой системы, инфекционно-воспалительные процессы в мочеполовом тракте, наличие инфекций, передаваемых половым путем, хронический алкоголизм, сексуальные дисфункции, гиперпродукция фолликулостимулирующего гормона.

Сбор и анализ эякулята осуществлялся в соответствии с критериями ВОЗ 2010 г. [14]. Анализ эякулята проводился при помощи микроскопа Micros Austria при увеличении 10×40. Сперматозоиды делились на группы в зависимости от их подвижности: сперматозоиды с прогрессивным движением $(\mathrm{A}+\mathrm{B})$, подвижные сперматозоиды $(\mathrm{A}+\mathrm{B}+\mathrm{C})$ и неподвижные сперматозоиды (D). Для определения жизнеспособности сперматозоидов использовалось суправитальное окрашивание по Блюму. Морфология сперматозоидов определялась в нативных препаратах, а также препаратах, окрашенных азур-эозином, на микроскопе Micros Austria при увеличении 10×100.

Оценку общей антиоксидантной активности проводили при помощи набора реагентов ОКСИСТАТ УП «ХОП ИБОХ НАН Беларуси». Концентрацию цинка определяли атомно-абсорбционным методом в спермоплазме.

Статистический анализ выполнен с помощью пакета прикладных программ «Statistica 6». Распределение полученных данных оказалось отличным от нормального, поэтому для оценки центральной тенденции использованы методы непараметрической статистики - медиана и квартили (Ме [q25; q75]). При корреляционном анализе использовалась корреляция по Спирмену. Для исследования различий признака в двух независимых группах использован критерий Манна-Уитни (U), для исследования различий в двух зависимых группах (повторных измерениях) использован критерий Вилкоксона (W). Нулевая гипотеза об отсутствии статистически значимых различий отвергалась при уровне статистической значимости $\mathrm{p}<0,05$.

\section{Результаты и обсуждение}

Контрольную группу составили 45 практически здоровых мужчин, имеющих детей в возрасте до 5 лет (доказанная фертильность). В группу исследования были включены 99 мужчин, имеющих жалобы на бесплодие в браке более 1 года и наличие олиго-, астено-, тератозооспермии либо их комбинацию в анализе эякулята (табл. 1).

Tаблица 1. - Антропометрическая характеристика мужчин, принявших участие в исследованиях, Ме [q25; q75]

\begin{tabular}{|c|c|c|c|}
\hline Показатель & $\begin{array}{c}\text { Контрольная } \\
\text { группа, } \mathrm{n}=45\end{array}$ & $\begin{array}{c}\text { Группа } \\
\text { иследования, } \\
\mathrm{n}=99\end{array}$ & $\begin{array}{c}\text { Статистическая } \\
\text { значимость } \\
\text { различий }\end{array}$ \\
\hline $\begin{array}{c}\text { Возраст, } \\
\text { годы }\end{array}$ & $\begin{array}{c}30,5 \\
{[28,0 ; 34,0]}\end{array}$ & $\begin{array}{c}32,0 \\
{[27,3 ; 34,0]}\end{array}$ & $\mathrm{p}=0,38$ \\
\hline ИМТ, кг/м² & $\begin{array}{c}23,3 \\
{[22,7 ; 24,5]}\end{array}$ & $\begin{array}{c}26,8 \\
{[24,8 ; 29,8]}\end{array}$ & $\mathrm{p}<0,001$ \\
\hline
\end{tabular}

Из представленной таблицы 1 видно, что мужчины двух групп относились к категории молодого возраста. Статистически значимых различий по возрасту между группами нами не выявлено. В группе исследования ИМТ был статистически значимо выше, причем число инфертильных мужчин с избыточной массой тела и ожирением составило 42,4 и 23,2\%, соответственно.

При оценке показателей в спермограмме в группе 1 и группе 2 установлены статистически значимые различия между двумя группами в жизнеспособности сперматозоидов, общем количестве сперматозоидов и их концентрации в эякуляте, общей и прогрессивной подвижности сперматозоидов, концентрации морфологически нормальных форм в сторону снижения показателей в группе исследования ( $<<0,001)$, при этом в показателях объема эякулята в двух группах статистически значимых различий нами не выявлено $(\mathrm{p}=0,57)$ (табл. 2).

Поскольку наличие окислительного стресса тесно связано с изменениями показателей эякулята: снижением концентрации, подвижности и жизнеспособности сперматозоидов, а также с ухудшением их морфологии, что постоянно подтверждается исследованиями, проведенными по данному поводу $[6,7,15]$, нами была проведена оценка общей антиоксидантной активности спермоплазмы у обследуемых в двух группах. Получены статистически значимые различия в общей антиоксидантной активности спермоплазмы между группой контроля и группой исследования $(\mathrm{p}<0,001)$. Кроме того, установлено, что на момент первичного обращения у 48,5\% 
Tаблица 2. - Показатели спермограммы у обследуемых мужчин, Me [q25; q75]

\begin{tabular}{|l|c|c|c|}
\hline \multicolumn{1}{|c|}{ Показатель } & $\begin{array}{c}\text { Контрольная } \\
\text { группа, } \mathrm{n}=45\end{array}$ & $\begin{array}{c}\text { Группа } \\
\text { исследования, } \\
\mathrm{n}=99\end{array}$ & $\begin{array}{c}\text { Статистическая } \\
\text { значимость } \\
\text { различий }\end{array}$ \\
\hline $\begin{array}{l}\text { Объем } \\
\text { эякулята, мл }\end{array}$ & $3,5[2,5 ; 4,5]$ & $3,2[2,8 ; 4,0]$ & $\mathrm{p}=0,57$ \\
\hline $\begin{array}{l}\text { Жизнеспособность } \\
\text { сперматозоидов, \% }\end{array}$ & 60,0 \\
{$[58,0 ; 70,0]$} & $\begin{array}{c}42,5 \\
{[24,0 ; 53,0]}\end{array}$ & $\mathrm{p}<0,001$ \\
\hline $\begin{array}{l}\text { Общее количество } \\
\text { сперматозоидов } \\
\text { в эякуляте, 10\% } \\
\text { эякулят }\end{array}$ & $\begin{array}{c}236,3[152,0 ; \\
300,0]\end{array}$ & $\begin{array}{c}36,0 \\
{[21,8 ; 51,9]}\end{array}$ & $\mathrm{p}<0,001$ \\
\hline $\begin{array}{l}\text { Концентрация } \\
\text { сперматозоидов в 1 } \\
\text { мл эякулята, 10 }\end{array}$ & {$[43,0 ; 98,5]$} & {$[8,0 ; 14,6]$} & $\mathrm{p}<0,001$ \\
\hline $\begin{array}{l}\text { Общая подвиж- } \\
\text { ность (А+В+С), \% }\end{array}$ & $60,0[55,0 ;$ & $\begin{array}{c}34 \\
{[21,5 ; 46,5]}\end{array}$ & $\mathrm{p}<0,001$ \\
\hline $\begin{array}{l}\text { Прогрессивная } \\
\text { подвижность, \% }\end{array}$ & $50,0[41,3 ;$ \\
\hline $\begin{array}{l}\text { Морфологически } \\
\text { нормальные } \\
\text { формы, \% }\end{array}$ & $\begin{array}{c}17.2[11,0 ; \\
{[10,5 ; 31,5]}\end{array}$ & $\begin{array}{c}7,0 \\
{[5,0 ; 12,0]}\end{array}$ & $\mathrm{p}<0,001$ \\
\hline
\end{tabular}

цинка и уровнем общей антиоксидантной активностью в эякуляте $(\mathrm{Rs}=0,38)$. Таким образом, определение концентрации цинка в спермоплазме у мужчин, страдающих бесплодием, и назначение препаратов на его основе с целью коррекции дефицита цинка является оправданным.

Учитывая полученные данные в группе исследования, всем пациентам была назначены лекарственные средства, обладающие антиоксидантными свойствами, на 3 месяца: L-карнитин - 500 мг 3 раза в день, витамин E - 400 мг по 1 капсуле 1 раз в день, цинк сульфат - 125 мг по 1 таблетке 2 раза в день, фолиевая кислота - 1 мг по 1 таблетке 1 раз в день, пентоксифиллин - 400 мг по 1 таблетке 1 раз в день.

Инфертильным мужчинам даны рекомендации по нормализации режима труда и отдыха, здоровому образу жизни. С целью контроля проводимого лечения через три месяца пациентам было рекомендовано сдать эякулят, в котором определялись основные параметры спермограммы, об- инфертильных пациентов антиоксидантная активность спермоплазмы находилась в пределах нормы, у 51,5\% - ниже 1,6 ммоль/л (рис. 1).

На основании этого было сделано предположение, что снижение общей антиоксидантной активности спермоплазмы и развитие окислительного стресса в сперме могут вносить существенный вклад в развитие мужской инфертильности.

Учитывая тот факт, что цинк играет важную роль в механизмах регуляции мужской репродуктивной системы [16], всем мужчинам из двух групп была определена концентрация цинка в спермоплазме.

Показатели концентрации цинка у фертильных и инфертильных мужчин представлены в таблице 3. Были отмечены статистически значимые различия в показателях значений концентрации цинка в двух группах $(\mathrm{p}<0.001)$. Кроме того, получены отрицательные корреляционные связи средней силы в группах между концентрацией цинка и концентрацией патологических форм в эякуляте $(\mathrm{Rs}=-0,32)$, а также положительные корреляционные связи между концентрацией

Tаблица 3. - Показатели антиоксидантной активности спермоплазмы и концентрации цинка в спермоплазме в обследуемых группах, Me [q25; q75]

\begin{tabular}{|c|c|c|c|c|}
\hline Показатель & $\begin{array}{c}\text { Референс- } \\
\text { ные } \\
\text { значения }\end{array}$ & $\begin{array}{c}\text { Контрольная } \\
\text { группа, } \mathrm{n}=45\end{array}$ & $\begin{array}{c}\text { Группа } \\
\text { исследования, } \\
\mathrm{n}=99\end{array}$ & $\begin{array}{c}\text { Статисти- } \\
\text { ческая } \\
\text { значимость } \\
\text { различий }\end{array}$ \\
\hline $\begin{array}{c}\text { Антиоксидантная } \\
\text { активность } \\
\text { спермоплазмы, } \\
\text { ммоль/л }\end{array}$ & $1,6-2,8$ & $\begin{array}{c}2,0 \\
{[1,9 ; 2,1]}\end{array}$ & $\begin{array}{c}1,7 \\
{[1,2 ; 1,9]}\end{array}$ & $\mathrm{p}<0,001$ \\
\hline $\begin{array}{c}\text { Концентрация цинка } \\
\text { в спермоплазме, } \\
\text { ммоль/л }\end{array}$ & $2,30-4,59$ & $\begin{array}{c}2,7 \\
{[2,46 ; 3,24]}\end{array}$ & $\begin{array}{c}1,62 \\
{[1,07 ; 2,0]}\end{array}$ & $\mathrm{p}<0,001$ \\
\hline
\end{tabular}

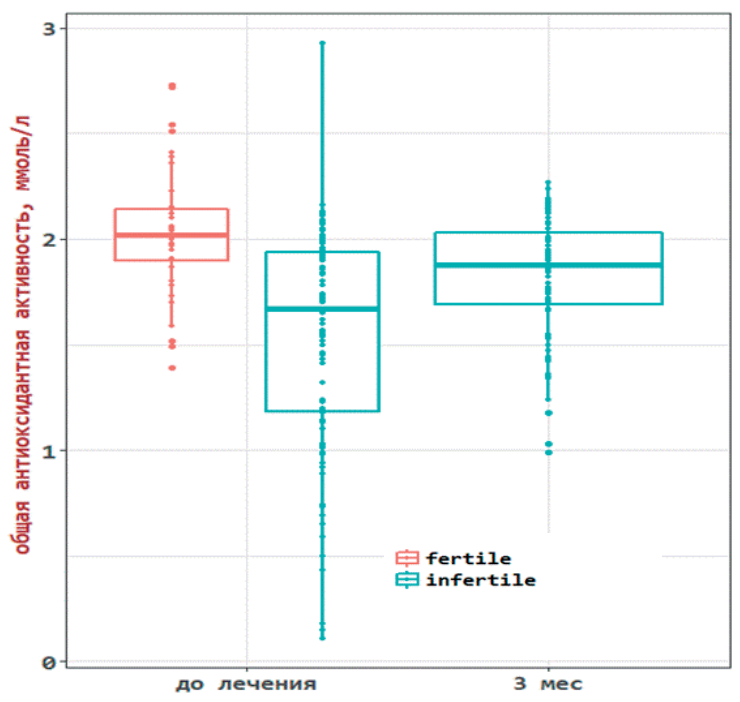

Рисунок 1. - Динамика изменений показателей общей антиоксидантной активности спермоплазмы на фоне проводимого лечения 
рез 3 месяца назначенного лечения достиг уровня 1,88 [1,69; 2,03] (рис. 1).

На фоне проводимого лечения произошло увеличение общей антиоксидантной активности спермоплазмы и уменьшилось воздействие окислительного стресса на сперматозоиды в яичках и придатках, что должно приводить к улучшению показателей спермограммы у пациентов, страдающих бесплодием. Такие выводы делают многие авторы $[4,7,10,11,17]$. Примером того является исследование Божедомова В. А и соавт., в котором показано, что прием антиоксидантов в течение 1,5 месяцев статистически значимо приводит к снижению выраженности окислительного стресса в эякуляте, о чем свидетельствует уменьшение АФК отмытыми сперматозоидами в 70\% случаев $(\mathrm{p}<0,05)$, и уменьшению концентрации сперматозоидов со смешанной патологией $(\mathrm{p}<0,01)$ [11].

При проведении анализа спермограмм инфертильных пациентов после «базисной» терапии нами установлено, что через 3 месяца лечения статистически значимо произошло увеличение значений показателей спермограммы (табл. 4). Наибольшая положительная динамика отмечена в показателе прогрессивной подвижности сперматозоидов (категория $\mathrm{A}+\mathrm{B}$ ) - увеличение на $44 \%$ от исходных значений, и концентрации морфологически нормальных форм - увеличение на 28,5\%. Кроме того, общая подвижность сперматозоидов увеличилась на 20,6\%, концентрация сперматозоидов в 1 мл эякулята - на 25\%, жизнеспособность - на 12,9\%.

Полученные данные согласуются с данными других авторов, показавших, что применение комплекса антиоксидантов в течение 3-х месяцев приводит к статистически значимым изменениям параметров спермограммы $[10,11]$. Так, Дендеберов Е. С. и соавт. в своем исследовании показали, что антиоксидантная терапия приводит к увеличению концентрации сперматозоидов в эякуляте на $18,5 \%$, общей подвижности сперматозоидов - на 33,7\%, активной подвижности сперматозоидов - на $38,4 \%$ и количества морфологически нормальных форм - на 50\% [11].

Tаблица 4. - Динамика показателей спермограммы инфертильных мужчин на фоне проводимого лечения, Ме [q25; q75]

\begin{tabular}{|c|c|c|c|}
\hline Показатель & До лечения & $\begin{array}{c}\text { После } \\
\text { лечения }\end{array}$ & $\begin{array}{c}\text { Статистическая } \\
\text { значимость }\end{array}$ \\
\hline $\begin{array}{c}\text { Концентрация } \\
\text { сперматозоидов в 1 мл } \\
\text { эякулята, 10 }\end{array}$ & $\begin{array}{c}12,0 \\
{[8,0 ; 14,6]}\end{array}$ & $\begin{array}{c}15,0 \\
{[11,6 ; 20,5]}\end{array}$ & $\mathrm{p}<0,05$ \\
\hline $\begin{array}{c}\text { Жизнеспособность } \\
\text { сперматозоидов, \% }\end{array}$ & $\begin{array}{c}42,5 \\
{[24,0 ; 53,0]}\end{array}$ & $\begin{array}{c}48,0 \\
{[36,0 ; 57,0]}\end{array}$ & $\mathrm{p}<0,001$ \\
\hline $\begin{array}{c}\text { Общая подвижность } \\
\text { (А+В+С), \% }\end{array}$ & $\begin{array}{c}34,0 \\
{[21,5 ; 46,5]}\end{array}$ & $\begin{array}{c}41,0 \\
{[28,0 ; 51,0]}\end{array}$ & $\mathrm{p}<0,001$ \\
\hline $\begin{array}{c}\text { Прогрессивная } \\
\text { подвижность, \% }\end{array}$ & $\begin{array}{c}18,0 \\
{[10,5 ; 31,5]}\end{array}$ & $\begin{array}{c}26,0 \\
{[13,0 ; 39,0]}\end{array}$ & $\mathrm{p}<0,001$ \\
\hline $\begin{array}{c}\text { Морфологически } \\
\text { нормальные формы, \% }\end{array}$ & $\begin{array}{c}7,0 \\
{[5,0 ; 12,0]}\end{array}$ & $\begin{array}{c}9,0 \\
{[6,0 ; 15,0]}\end{array}$ & $\mathrm{p}<0,001$ \\
\hline
\end{tabular}

Однако нами отмечено, что даже после проводимого лечения у большинства пациентов показатели спермограммы находились ниже референсных значений, установленных ВО3 (2010) [14]. Так, у 59,5\% мужчин концентрация сперматозоидов в 1 мл эякулята была ниже $15 \times 106 /$ эякулят, у 62,6\% прогрессивная подвижность сперматозоидов находилась ниже 32\% (категория сперматозоидов $\mathrm{A}+\mathrm{B})$, а у $44 \%$ общая подвижность сперматозоидов ниже 40\% (категория сперматозоидов $\mathrm{A}+\mathrm{B}+\mathrm{C})$, у 78,8\% пациентов концентрация жизнеспособных сперматозоидов была ниже $58 \%$. В основном отсутствие статистически значимого улучшения $(\mathrm{p}>0,05)$ в показателях спермограммы (концентрация сперматозоидов в эякуляте, прогрессивная и общая подвижность сперматозоидов) не было у мужчин с избыточной массой тела, концентрацией тестостерона в сыворотке крови ниже 12 нмоль/л, гипергликемией и дислипидемией.

В связи с этим нами сделано предположение, что прием лекарственных средств, способствующих улучшению параметров спермограммы, в течение трех месяцев не является достаточным, необходимо проведение дальнейшего этиопатогенетически обоснованного лечения в зависимости от выявленных нарушений.

При оценке динамики показателей концентрации цинка после проведенного лечения отмечено статистически значимое увеличение концентрации цинка в спермоплазме у инфертильных пациентов ( $<00,001)$ (рис. 2). Медиана концентрации цинка определялась на уровне $2,08[1,51 ; 2,58]$ ммоль/л (рис. 2). Были установлены положительные корреляционные связи между увеличением концентрации цинка в эякуляте и общей антиоксидантной активностью $(\mathrm{Rs}=0,26)$, а также увеличением концентрации цинка и концентрацией сперматозоидов в 1 мл эякулята $(\mathrm{Rs}=0,3)$.

Схожие данные получены многими авторами $[18,19]$. Так, в метаанализе, проведенном Taravati A. et al., было показано, что снижение концентрации цинка в эякуляте отрицательно коррелирует с показателями спермограммы у мужчин, страдающих бесплодием. Применение препаратов на основе цинка приводит к статистически значимому улучшению прогрессивной подвижности сперматозоидов и их концентрации в эякуляте $(p=0,044)$ [19]. Неймарк А. И. и соавт. использовали оригинальный препарат для лечения патоспермии на основе цинка в течение трех месяцев. После проведенного лечения в анализе эякулята инфертильных мужчин отмечалось увеличение концентрации живых сперматозоидов на 28\%, увеличение прогрессивной подвижности на $39 \%$, количество морфологически нормальных форм увеличилось на $24 \%$, у 26\% пар, проходивших лечение, наступила беременность у партнерш [18].

В свою очередь исследование Оmu A. et al. показало, что монотерапия 


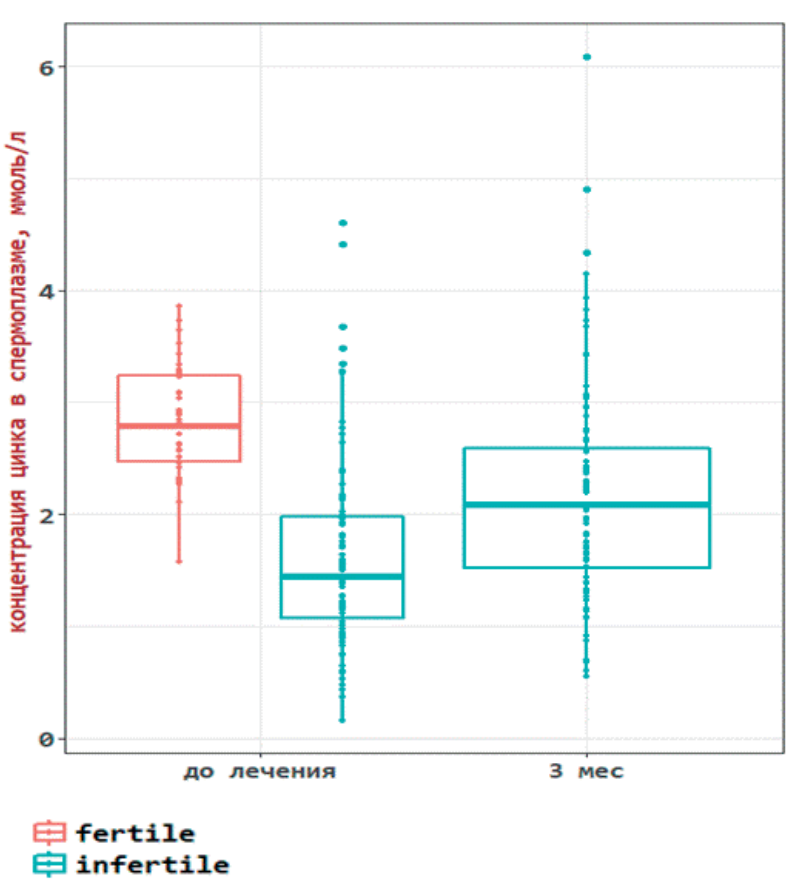

Рисунок 2. - Динамика изменения концентрации цинка в спермоплазме на фоне проводимого лечения

мужского бесплодия препаратами цинка не приводит к статистически значимым изменениям в анализах эякулята по сравнению с тем, если использовать цинк в комбинации с антиоксидантами - витамином Е и $\mathrm{C}(\mathrm{p}<0,05)[20]$.

Таким образом, полученные в исследовании результаты требуют дальнейшего изучения и анализа, а также служат важным шагом к пониманию причин мужского бесплодия и могут

\section{Лumepamypa}

1. Guideline on male infertility / A. Jungwirth [et al.]. London : EAU, 2015. $-42 \mathrm{p}$.

2. Актуальные вопросы оказания помощи парам с мужским фактором бездетного брака: клинические и организационно-методические аспекты / В. А. Божедомов [и др.] // Андрология и генитальная хирургия. - 2013. - Т. 14, № 4. - С. 7-16. doi: 10.17650/2070-9781-2013-4-7-16.

3. Сухих, Г. Т. Мужское бесплодие : новейшее руководство для урологов и гинекологов / Г. Т. Сухих, В. А. Божедомов. - Москва : Эксмо, 2009. - 240 с.

4. Божедомов, В. А. Мужской фактор бездетного брака - пути решения проблемы / В. А. Божедомов // Урология. - 2016. - № 1. - С. 28-34.

5. Мсхалая, Г. Ж. Мужское бесплодие. State of art Г. Ж. Мсхалая [и др.]. - Москва : Практическая медицина, 2014. $-80 \mathrm{c}$.

6. Are oxidative stress markers associated with unexplained male infertility? / B. J. Mayorga Torres [et al.] // Andrologia. - 2017. - Vol. 49, № 5. - doi: 10.1111/ and.12659.

7. Oxidative stress and medical antioxidant treatment in male infertility / F. M. Lanzafame [et al.] // Reproductive biomedicine online. - 2009. - Vol. 19, № 5. - P. 638-659.

8. Effect of oxidative stress on male reproduction / A. Agarwal [et al.] // World Journal Men's Health. - 2014.

-Vol. 32, № 1.-P. 1-17. - doi: 10.5534/wjmh.2014.32.1.1. быть использованы для разработки патогенетически обоснованных методов его коррекции.

\section{Выводы}

1. У мужчин, страдающих бесплодием, по сравнению с фертильными мужчинами отмечается снижение общей антиоксидантной активности спермоплазмы и концентрации цинка в сперме. Снижение общей антиоксидантной активности может являться фактором развития мужского бесплодия.

2. Назначение комплекса лекарственных средств в составе L-карнитина, пентоксифиллина, цинк сульфата, витамина Е, фолиевой кислоты сроком на три месяца улучшает общую антиоксидантную активность спермоплазмы, а также качественные и количественные характеристики эякулята у инфертильных мужчин.

3. У инфертильных пациентов с избыточной массой тела, гипергликемией, дислипидемией и андрогенным дефицитом по сравнению с мужчинами, у которых не выявлено подобных нарушений, применение антиоксидантов не приводит к статистически значимым изменениям в параметрах спермограммы ( $>00,05)$.

4. Поскольку применение антиоксидантов в течение трех месяцев у мужчин с избыточной массой тела, гипергликемией, дислипидемией, андрогенным дефицитом не приводит к статистически значимому улучшению концентрации сперматозоидов в эякуляте, прогрессивной и общей подвижности сперматозоидов, данной группе пациентов необходимо проведение дальнейшего этиопатогенетически обоснованного лечения в зависимости от выявленных у них нарушений.

9. Acrosome Reaction as a Preparation for Gamete Fusion / P. S. Cuasnicú [et al.] // Sperm Acrosome Biogenesis and Function During Fertilization / ed.: M. G. Buffone. - London, 2016. - P. 159-172. - doi: 10.1007/978-3-31930567-7 9 .

10. Ефремов, Е. А. Антиоксидантная терапия мужского бесплодия как возможность улучшить исходы вспомогательных репродуктивных технологий / Е. А. Ефремов, Е. В. Касатонова, Я. И. Мельник // Эффективная фармакотерапия. - 2017. - № 22. C. 32-43.

11. Применение комплекса нутриентов для лечения мужского бесплодия / В. А. Божедомов [и др.] // Русский медицинский журнал. - 2016. - Т. 24, № 23. - C. 1546-1552.

12. Antioxidants for male subfertility [Electronic resource]/ M. G. Showell [et al.] // Cochrane Library. - 2013. - Vol. 8. - Mode of access: https://www.cochranelibrary.com/ cdsr/doi/10.1002/14651858.CD007807.pub2./full. - Data of access: 20.09.2018.

13. Moslemi, M. K. Selenium - vitamin E supplementation in infertile men: effects on semen parameters and pregnancy rate / M. Moslemi, S. Tavanbakhsh // International journal of general medicine. - 2011. - Vol. 4. - P. 99. doi: 10.2147/IJGM.S16275.

14. World Health Organization reference values for human semen characteristics / T. G. Cooper [et al.] // Human Reproduction Update. - 2010. - Vol. 16, № 3. - P. 231-245. 
15. Mechanisms of oligozoospermia: an oxidative stress perspective / A. Agarwal [et al.] // Systems Biology in Reproductive Medicine. - 2014. - Vol. 60, № 4. - P. 206216.

16. Brody, S. А. Мужское бесплодие и окислительный стресс: роль диеты, образа жизни и пищевых добавок / S. A. Brody // Андрология и генитальная хирургия. - 2014. - T. 15, № 3. - C. 33-41. - doi: 1017650/20709781-2014-3-33-41.

17. Majzoub, A. Antioxidant therapy in idiopathic oligoasthenoteratozoospermia / A. Majzoub, A. Agarwal // Indian Journal of Urology. - 2017. - Vol. 33, № 3. - P. 207-214. - doi: 10.4103 /iju.IJU_15_17.

18. Неймарк, А. И. Применение препарата «Селцинк плюс» у мужчин с нарушением фертильности / А. И. Неймарк, И. И. Клепикова // Андрология и генитальная хирургия. - 2013. - № 4. - С. 77-80.

19. Taravati, A. Association between seminal plasma zinc level and asthenozoospermia: a meta-analysis study / A. Taravati, F. Tohidi // Andrologia. - 2016. - Vol. 48, № 6. - P. 646-653. - doi: 10.1111/and.12494.

20. Indications of the mechanisms involved in improved sperm parameters by zinc therapy / A. E. Omu [et al.] // Medical Principles and Practice. - 2008. - Vol. 17, № 2. P. 108-116. - doi: 10.1159/000112963.

\section{Reference}

1. Jungwirth A, Diemer T, Dohle GR, Giwercman A, Kopa Z, Krausz C, Tournaye H. Guideline on male infertility. London: EAU; 2015. 42p.

2. Bozhedomov VA, Rohlikov IM, Tretjakov AA, Lipatova NA, Vinogradov IV, Nikonov EL. Aktualnye voprosy okazanija pomoshhi param s muzhskim faktorom bezdetnogo braka: klinicheskie i organizacionno-metodicheskie aspekty [Topical issues of rendering assistance to couples with the male factor of childless marriage: clinical and organizational-methodical aspects]. Andrologija $i$ genital'naja hirurgija [Andrology and Genital Surgery]. 2013;14(4):7-16. doi: 10.17650/2070-9781-2013-4-7-16. (Russian).

3. Suhih GT, Bozhedomov VA. Muzhskoe besplodie [Male infertility]. Moskva: Jeksmo; 2009. 240 p. (Russian).

4. Bozhedomov VA. Muzhskoj faktor bezdetnogo braka - puti reshenija problemy [The male factor of childless marriage is the way to solve the problem]. Urologija [Urology]. 2016;1:28-34. (Russian).

5. Mshalaja GZh, Kalichenko SJu, Tishova JuA. Muzhskoe besplodie. State of art [Male infertility. State of art]. Moskva: Prakticheskaja medicina; 2014. 80 p. (Russian).

6. Mayorga Torres BJM, Camargo M, Cadavidi AP, du Plessis SS, Cardona Maya WD. Are oxidative stress markers associated with unexplained male infertility? Andrologia. 2017;49(5). doi: 10.1111/and.12659.

7. Lanzafame FM, La Vignera S, Vicari E, Calogero AE. Oxidative stress and medical antioxidant treatment in male infertility. Reproductive biomedicine online. 2009;19(5):638-659.

8. Agarwal A, Virk G, Ong C, du Plessis SS. Effect of oxidative stress on male reproduction. World Journal Men's Health. 2014;32(1):1-17. doi: 10.5534/wjmh.2014.32.1.1.
9. Cuasnicú PS, Da Ros VG, Munoz MW, Cohen DJ. Acrosome Reaction as a Preparation for Gamete Fusion. In: Buffone MG, editors. Sperm Acrosome Biogenesis and Function During Fertilization. London: Springer; 2016. p. 159-172. doi: 10.1007/978-3-319-30567-7 9.

10. Efremov EA, Kasatonova EV, Melnik JaI. Antioksidantnaja terapija muzhskogo besplodija kak vozmozhnost' uluchshit ishody vspomogatelnyh reproduktivnyh tehnologij [Antioxidant therapy of male infertility as an opportunity to improve outcomes of assisted reproductive technologies]. Jeffektivnaja farmakoterapija. 2017;22:32-43. (Russian).

11. Bozhedomov VA, Lipatova NA, Bozhedomova GE, Shherbakova EV, Komarina RA. Primenenie kompleksa nutrientov dlja lechenija muzhskogo besplodija [Application of a complex of nutrients for the treatment of male infertility]. Russkij medicinskij zhurnal [Russian Medical Journal]. 2016;24(23):1546-1552. (Russian).

12. Showell MG, Brown J, Clarke J, Hart RJ. Antioxidants for male subfertility [Internet]. Cochrane Database of Systematic Reviews. 2013;8. Available from: https:// www.cochranelibrary.com/cdsr/doi/10.1002/14651858. CD007807.pub2/full.

13. Moslemi M, Tavanbakhsh S. Selenium - vitamin E supplementation in infertile men: effects on semen parameters and pregnancy rate. International journal of general medicine. 2011;4:99. doi: 10.2147/IJGM.S16275.

14. Cooper TG, Noonan E, von Eckardstein S, Auger J, Cordon Baker HW, Behre HM, Haugen TB, Krugen T, Wang C, Mbizvo MT, Vogelsong KM. World Health Organization reference values for human semen characteristics. Human Reproduction Update. 2010;16(3):231-245.

15. Agarwal A, Mulgund A, Sharma R, Sabanegh E. Mechanisms of oligozoospermia: an oxidative stress perspective. Systems Biology in Reproductive Medicine. 2014;60(4):206-216.

16. Brody SA. Muzhskoe besplodie i okislitelnyj stress: rol diety, obraza zhizni i pishhevyh dobavok [Male infertility and oxidative stress: the role of diet, lifestyle and nutritional supplements]. Andrologija i genitalnaja hirurgija [Andrology and Genital Surgery]. 2014;15(3):33-41. doi: 1017650/2070-9781-2014-3-33-41. (Russian).

17. Majzoub A, Agarwal A. Antioxidant therapy in idiopathic oligoasthenoteratozoospermia. Indian Journal of Urology. 2017;33(3):207-214. doi: 10.4103 /iju.IJU_15_17.

18. Nejmark AI, Klepikova II. Primenenie preparata «Selcink pljus» u muzhchin s narusheniem fertilnosti [The use of «Selzinc plus» in men with impaired fertility]. Andrologija i genitalnaja hirurgija [Andrology and Genital Surgery]. 2013;4:77-80. (Russian).

19. Taravati A, Tohidi F. Association between seminal plasma zinc level and asthenozoospermia: a meta-analysis study. Andrologia. 2016;48(6):646-653. doi: 10.1111/ and.12494.

20. Omu AE, Al-Azemi MK, Kehinde EO, Anim JT, Oriowo MA, Mathew TC. Indications of the mechanisms involved in improved sperm parameters by zinc therapy. Medical Principles and Practice. 2008;17(2):108-116. doi: 10.1159/000112963. 


\title{
INFLUENCE OF ANTIOXIDANTS ON THE PARAMETERS OF SPERMOGRAM IN INFERTILE MEN
}

\author{
Nitkin D. M., Rakevich M. V., Zhurko P. T.
}

State Educational Institution "Belarusian Medical Academy of Postgraduate Education", Minsk, Belarus

Background. The development of methods for diagnosis and treatment of male infertility is a topical issue nowadays.

The aim of the study was to determine the spermogram index, the antioxidant capacity of spermoplasm and the concentration of zinc in the sperm of healthy fertile and infertile males as well as to evaluate the effects of drugs with antioxidant activity on the parameters of the spermogram in infertile men.

Material and methods. A total of 144 males of reproductive age participated in the study: 45 fertile and 99 infertile ones. Collection and analysis of the ejaculate was carried out in accordance with WHO criteria (2010).

Results. There were statistically significant differences in the parameters of the spermogram between fertile and infertile men $(p<0.05)$. In $48.5 \%$ of infertile patients the antioxidant activity of the spermoplasm was normal, and in $51.5 \%$ of males it was below $1.6 \mathrm{mmol} / \mathrm{L}$. The concentration of zinc in men with infertility averaged 1.62 [1.07, 2.0] mmol/L. The use of the complex of antioxidants composed of L-carnitine, pentoxifylline, zinc sulfate, vitamin E, folic acid for a period of 3 months improved the qualitative and quantitative characteristics of the ejaculate in infertile men $(p<0.05)$, as well as the overall antioxidant activity of the spermoplasm $(1.88$ [1.69, 2.03] mmol/L) and the concentration of zinc in the sperm $(2.08[1.51,2.58] \mathrm{mmol} / \mathrm{L})$.

Conclusions. Reduction of the total antioxidant activity is a factor in the development of male infertility. Prescription of the complex of antioxidants improves the qualitative and quantitative indices of the spermogram.

Keywords: male infertility, spermogram, antioxidant activity of spermoplasm, treatment of infertility.

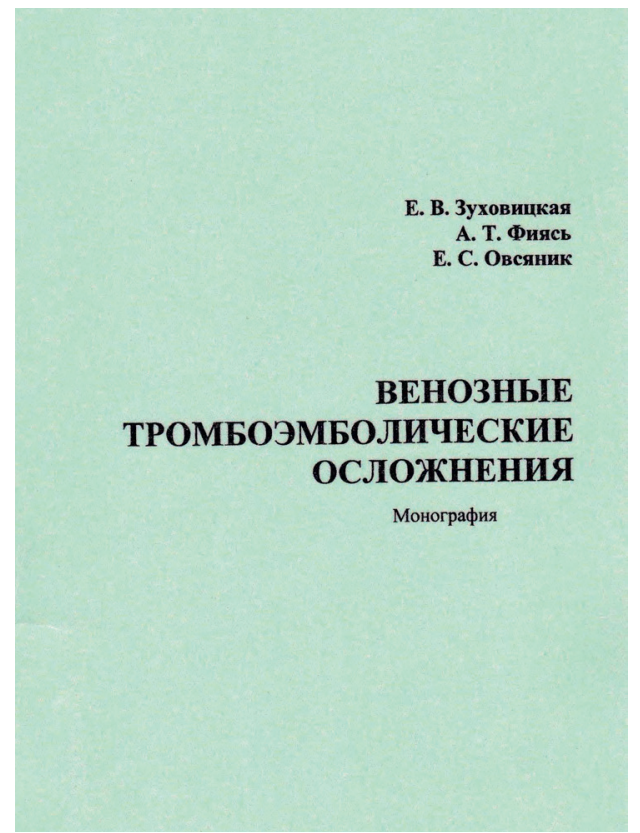

Зуховицкая, Елена Владимировна.

Венозные тромбоэмболические осложнения : монография / Е. В. Зуховицкая, А. Т. Фиясь, Е. С. Овсяник ; Министерство здравоохранения Республики Беларусь, Учреждение образования "Гродненский государственный медицинский университет", 1-я кафедра внутренних болезней. - Гродно : ГрГМУ, 2018. - 230 с. : табл., рис. - Библиогр.: с. 220-228. - ISBN 978985-558-975-5.

В монографии изложены современные данные по основам гемостаза в норме и при патологии. Представлены принципь сосудисто-тромбочитарного и коагуляиионного гемостаза в норме и при различных патологических состояниях. Указаны причины развития венозного тромбоэмболизма в клинической практике в разных группах паичентов, методы обследования и диагностики. Приведены рекомендации по обследованию и проведению антикоагулянтной терапии при осложнениях венозного тромбоэмболизма согласно последним данным ведущзих клинических учреждений.

Монография предназначена для руководства в практической работе врачам-гематологам, хирургам, акушерам-гинекологам, а также врачам общей практики. 